\title{
Towards a non-perturbative measurement of the heavy quark momentum diffusion coefficient
}

\author{
A. Francis, O. Kaczmarek, M. Laine, J. Langelage* \\ Faculty of Physics, University of Bielefeld, D-33501 Bielefeld, Germany \\ E-mail: afrancis, okacz, laine, jlangephysik.uni-bielefeld.de
}

\begin{abstract}
We report on a lattice investigation of heavy quark diffusion within pure $\mathrm{SU}(3)$ plasma above the deconfinement transition, with the quarks treated to leading order in the heavy mass expansion. Using a multilevel algorithm, several volumes and lattice spacings, as well as tree-level improvement and perturbative renormalization, we measure the relevant "colour-electric" Euclidean correlator, finding that it clearly exceeds its perturbative counterpart. Even without analytic continuation, this suggests that at temperatures just above the critical one, non-perturbative interactions felt by the heavy quarks are stronger than within the weak-coupling expansion. After introducing rough modelling of the spectral shape, diffusion coefficients down to $D \sim 0.5 / T$ appear possible.
\end{abstract}

The XXIX International Symposium on Lattice Field Theory - Lattice 2011

July 10-16, 2011

Squaw Valley, Lake Tahoe, California

\footnotetext{
* Speaker.
} 


\begin{tabular}{|c|c|c|c|c|}
\hline$T / T_{\mathrm{c}}$ & $\beta$ & $N_{\tau}$ & $N_{s}$ & $N_{\text {conf }}$ \\
\hline 1.5 & 6.872 & 16 & 32 & 200 \\
& & & 48 & 75 \\
& & & 64 & 50 \\
& 7.192 & 24 & 64 & 338 \\
2.25 & 7.192 & 16 & 48 & 125 \\
& & & 64 & 50 \\
& 7.457 & 24 & 64 & 504 \\
\hline
\end{tabular}

\begin{tabular}{|c|c|c|c|c|}
\hline$T / T_{\mathrm{c}}$ & $\beta$ & $N_{\tau}$ & $N_{s}$ & $N_{\text {conf }}$ \\
\hline 3.0 & 7.457 & 16 & 48 & 127 \\
& & & 64 & 50 \\
& 7.793 & 24 & 64 & 469 \\
$\gg 1$ & 8.426 & 24 & 64 & 44 \\
& 9.794 & & & 51 \\
& 20.0 & & & 51 \\
& 30.0 & & & 11 \\
\hline
\end{tabular}

Table 1: Overview of the parameter values simulated and statistics accumulated $\left(\beta=2 N_{\mathrm{c}} / g^{2}\right)$.

\section{Introduction}

As was previously observed at the RHIC and recently confirmed by the heavy ion program at the LHC [1], jets containing $c$ or $b$ quarks ( $D$ or $B$ mesons) get effectively "quenched", meaning that they experience a rapid kinetic equilibration with the thermal medium generated in the collision. The rate at which this happens appears to be much faster than at leading order of the weak-coupling expansion [2]. A next-to-leading order analysis suggests that indeed there are large corrections from higher orders [3], underlining the importance of a non-perturbative study. For the charm quark a direct measurement of the heavy quark diffusion coefficient from the current-current correlator is under way [ [ $[$ ] (with techniques developed for light quarks in ref. [5]), but given the systematic uncertainties involved as well as the fact that the bottom quark case is also of interest, it appears worthwhile to contrast this with an alternative approach, based on Heavy Quark Effective Theory (HQET) and valid in the large-mass limit [6]. Following a previous investigation, which demonstrated the principal applicability of the method [ [7], we report here on progress towards a removal of lattice artifacts and a physical interpretation of the results.

\section{Observable}

Heavy quarks carry a colour charge and, whenever there are gauge fields present, are therefore subject to a coloured Lorentz force. Like with other transport coefficients the corresponding "lowenergy constants" are easiest to define at vanishing three-momentum; then the Lorentz-force is proportional to the electric field strength. This leads to a "colour-electric correlator" [8, 6],

$$
G_{\mathrm{E}}(\tau) \equiv-\frac{1}{3} \sum_{i=1}^{3} \frac{\left\langle\operatorname{Re} \operatorname{Tr}\left[U\left(\frac{1}{T} ; \tau\right) g E_{i}(\tau, \mathbf{0}) U(\tau ; 0) g E_{i}(0, \mathbf{0})\right]\right\rangle}{\left\langle\operatorname{Re} \operatorname{Tr}\left[U\left(\frac{1}{T} ; 0\right)\right]\right\rangle}
$$

where $g E_{i}$ denotes the colour-electric field, $T$ the temperature, and $U\left(\tau_{2} ; \tau_{1}\right)$ a Wilson line in Euclidean time direction. If the corresponding spectral function, $\rho_{\mathrm{E}}$, can be extracted [9], then the "momentum diffusion coefficient", often denoted by $\kappa$, can be obtained from

$$
\kappa=\lim _{\omega \rightarrow 0} \frac{2 T \rho_{\mathrm{E}}(\omega)}{\omega}
$$

According to non-relativistic linear response relations (valid for $M \gg \pi T$, where $M$ stands for a heavy quark pole mass) the corresponding "diffusion coefficient" is given by $D=2 T^{2} / \kappa$. 

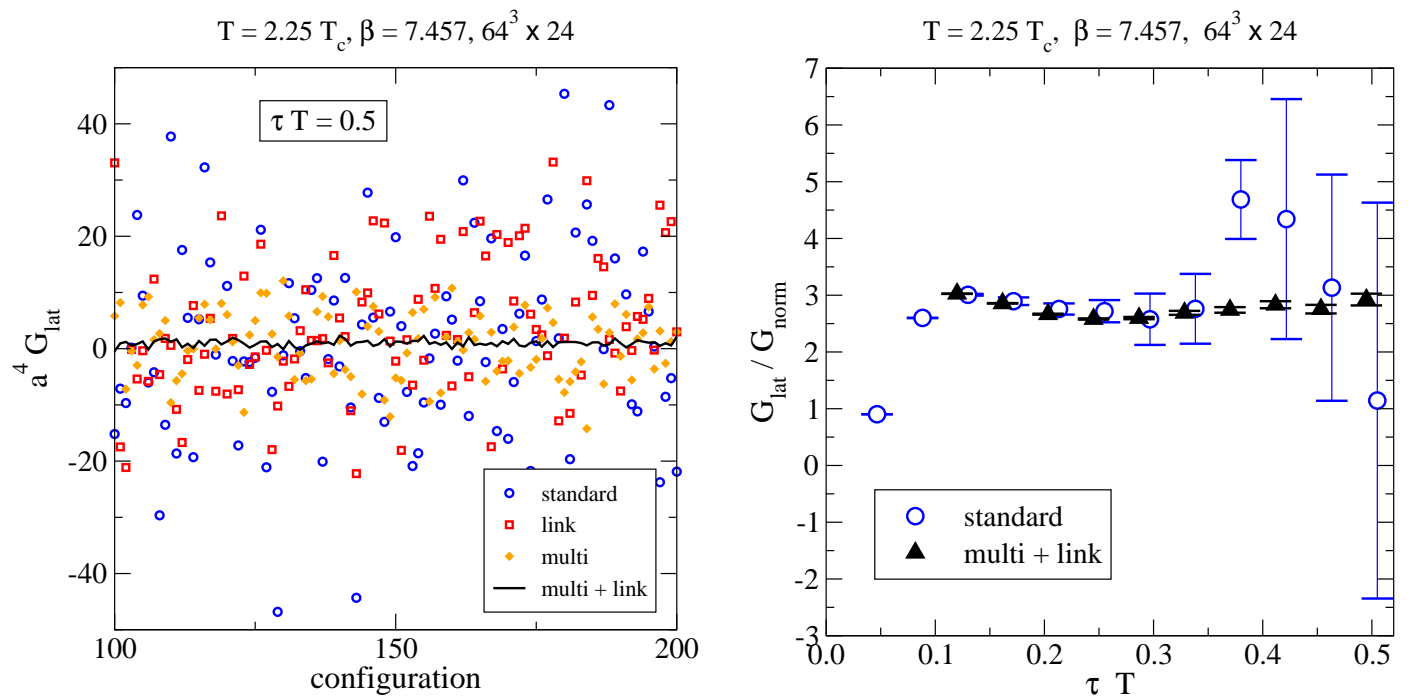

Figure 1: Left: Part of a representative Monte Carlo history. Right: The corresponding averages.

\section{How to get a signal}

In order to carry out a measurement (our parameter values, referring to the standard Wilson gauge action, are listed in table 1), eq. (2.1) needs to be discretized; we follow the proposal of ref. [6], viz.

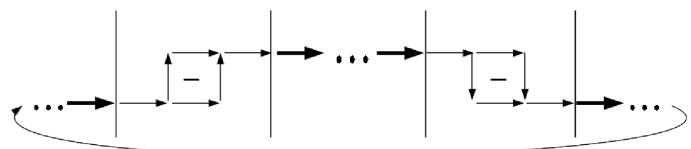

We make use of two special techniques: the "thick" links in between the electric fields are handled through the link integration method [10, 11], whereas the time intervals of width $3 a$, enclosing the electric fields, are subjected to $\sim 10$ extra updates with fixed boundary conditions, according to the multilevel philosophy [12] (a previous application at finite temperatures can be found e.g. in ref. [13]). It depends on the parameters which of the two techniques helps more, but when both are combined, we always get a signal; this is illustrated in fig. 1. In the right panel, the results have been normalized through [6]

$$
G_{\text {norm }}(\tau T) \equiv \frac{G_{\text {cont }}^{\mathrm{LO}}(\tau T)}{g^{2} C_{F}}=\pi^{2} T^{4}\left[\frac{\cos ^{2}(\pi \tau T)}{\sin ^{4}(\pi \tau T)}+\frac{1}{3 \sin ^{2}(\pi \tau T)}\right], \quad C_{F} \equiv \frac{N_{\mathrm{c}}^{2}-1}{2 N_{\mathrm{c}}} .
$$

\section{Calibration and volume dependence}

In order to crosscheck the code, as well as for later reference, we have computed the correlator of eq. (3.1) to leading order in lattice perturbation theory. The result reads

$$
\begin{aligned}
& G_{\text {lat }}^{\mathrm{LO}}(\tau T)=\frac{g^{2} C_{F}}{3 a^{4}} \int_{-\pi}^{\pi} \frac{\mathrm{d}^{3} \mathbf{q}}{(2 \pi)^{3}} \frac{\mathrm{e}^{\bar{q} N_{\tau}(1-\tau T)}+\mathrm{e}^{\bar{q} N_{\tau} \tau T}}{\mathrm{e}^{\bar{q} N_{\tau}}-1} \frac{\tilde{q}^{2}}{\sinh \bar{q}}, \\
& \bar{q} \equiv 2 \operatorname{arsinh}\left(\frac{\tilde{q}}{2}\right), \quad \tilde{q}^{2} \equiv \sum_{i=1}^{3} 4 \sin ^{2}\left(\frac{q_{i}}{2}\right), \quad \tilde{Q}^{2} \equiv \sum_{i=1}^{4} 4 \sin ^{2}\left(\frac{q_{i}}{2}\right),
\end{aligned}
$$



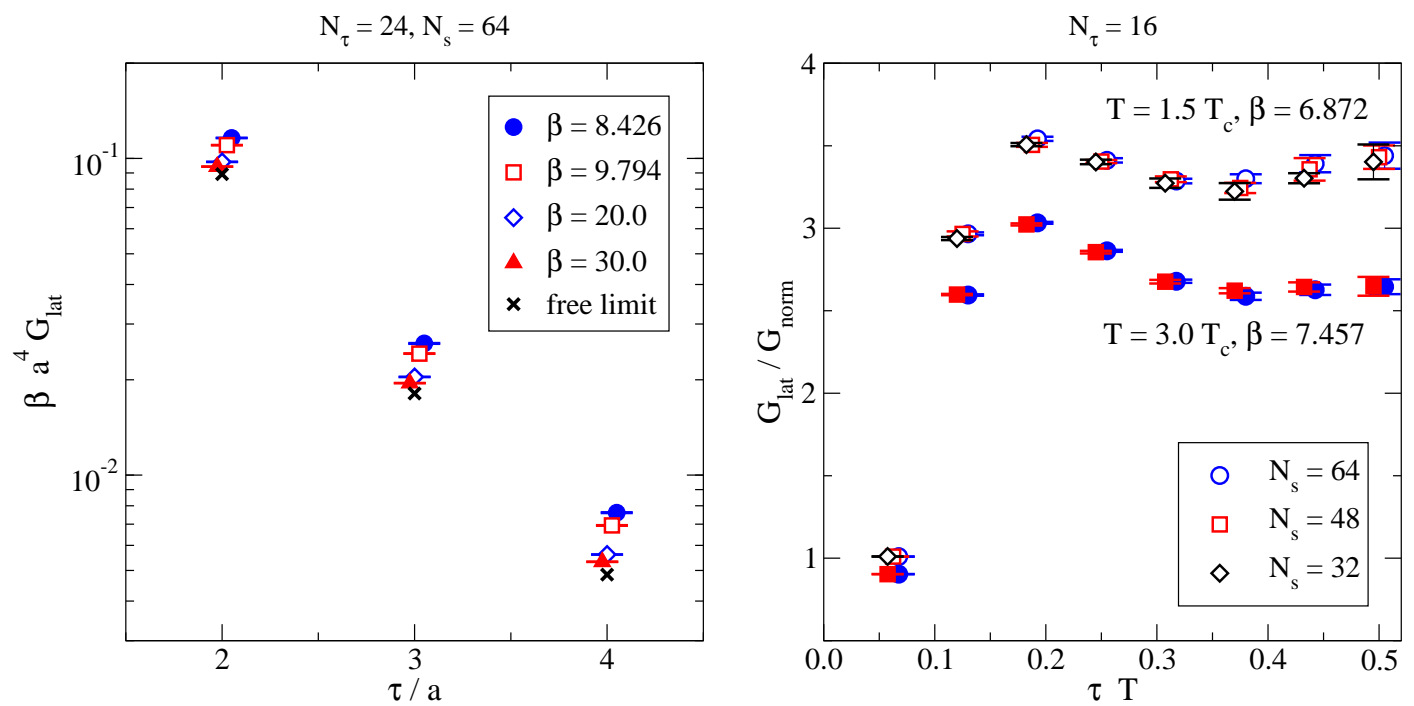

Figure 2: Left: A crosscheck in the weak-coupling limit. Right: Volume dependence at two temperatures.

which for $N_{\tau} \rightarrow \infty, a \rightarrow 0$ goes over into the continuum result $G_{\text {cont }}^{\mathrm{LO}}(\tau T)$ of eq. (3.2). In fig. 2(left) a comparison of eq. (4.1) with lattice measurements at very large values of $\beta=2 N_{\mathrm{c}} / g^{2}$ is shown, and we observe agreement in the weak-coupling limit.

Proceeding towards physical measurements, fig. 2(right) shows results at various spatial volumes. At the current level of resolution, finite-volume effects are seen to be below statistical errors.

\section{Discretization effects}

An important systematic error originates from a finite lattice spacing $(a \neq 0)$. A memory limitation currently prohibits us from increasing the spatial volume beyond $64^{3}$, but in view of the very small volume-dependence seen in fig. 2(right), a comparison of the lattices $48^{3} \times 16$ and $64^{3} \times 24$ (the latter approximating the desired $72^{3} \times 24$ ) at the same temperature allows us to test for the existence of scaling violations. Results at two different temperatures are shown in fig. B(left), and it is clear that effects related to a finite lattice spacing need to be brought under control.

Fortunately, the situation can be all but rectified through "tree-level improvement" [14, 15]. Using eqs. (3.2), (4.1) to determine $\overline{\tau T}$ from

$$
G_{\text {cont }}^{\mathrm{LO}}(\overline{\tau T})=G_{\text {lat }}^{\mathrm{LO}}(\tau T),
$$

the pairs $(\tau T, \overline{\tau T})$ allow us to define the tree-level improved data from the measured correlator $G_{\text {lat }}(\tau T)$ according to

$$
G_{\mathrm{imp}}(\overline{\tau T}) \equiv G_{\mathrm{lat}}(\tau T) .
$$

The results are shown in fig. 3(right) and look much nicer than those in fig. 3(left).

There are discretization effects at loop levels as well. Following the general Symanzik philosophy [16] as well as the derivation of the colour-electric correlator in ref. [6], we may expect that the renormalization factor is related to the coefficient of the kinetic energy operator in lattice HQET, 

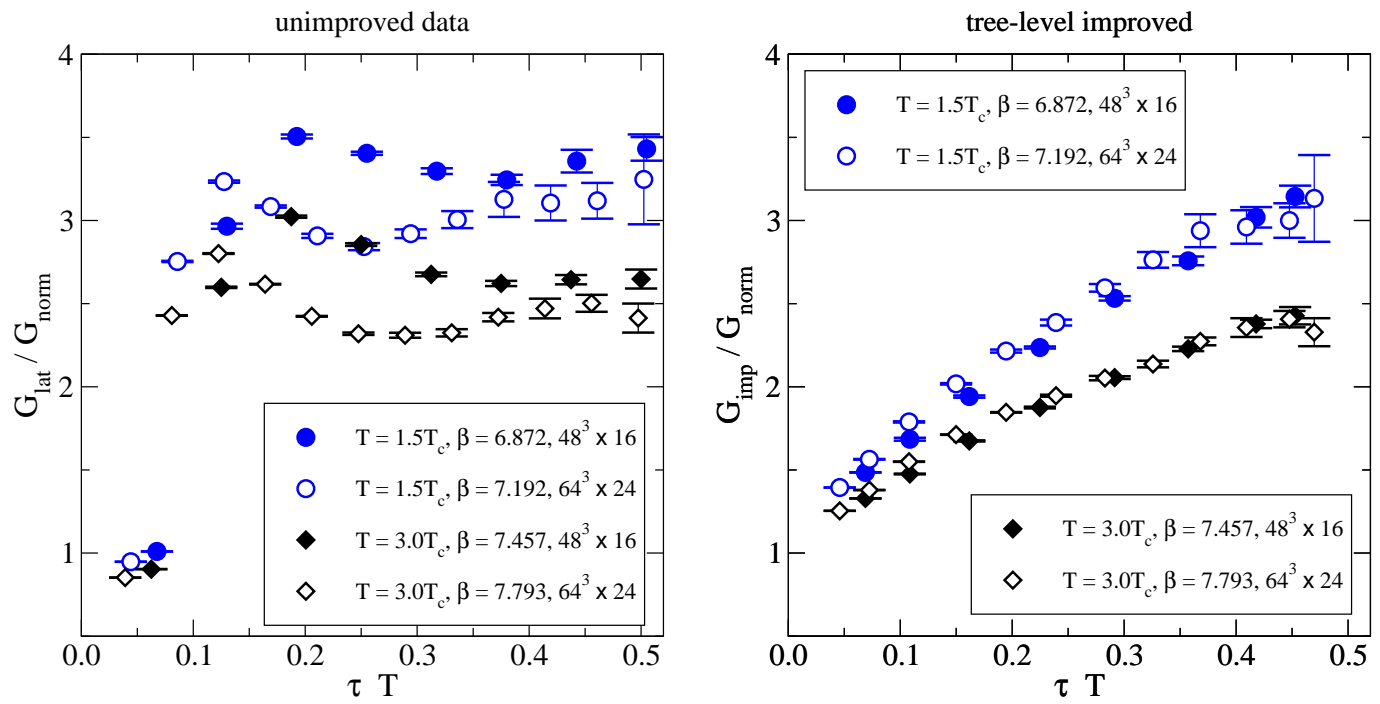

Figure 3: Lattice spacing dependence at fixed $T$ without (left) and with (right) tree-level improvement.

let us denote it by $c_{2}$, which needs to be appropriately tuned to match to continuum. In perturbation theory the value can be determined by computing the heavy quark self-energy and choosing $c_{2}$ so as to cancel 1-loop effects specific to lattice regularization. Making use of techniques introduced in ref. [17], we find

$$
c_{2} \approx 1-\frac{g^{2} C_{F}}{2}\left\{\int_{-\pi}^{\pi} \frac{\mathrm{d}^{3} \mathbf{q}}{(2 \pi)^{3}} \frac{1}{\tilde{q}^{2}}-\frac{2}{3} \int_{-\pi}^{\pi} \frac{\mathrm{d}^{4} \mathbf{q}}{(2 \pi)^{4}} \frac{1}{\tilde{Q}^{2}}\right\} \approx 1-\frac{0.59777}{\beta},
$$

with $\tilde{q}^{2}, \tilde{Q}^{2}$ defined in eq. (4.2). The renormalization factor is then $c_{2}^{2} \approx 0.83 \ldots 0.85$, i.e. quite modest. The results obtained after this correction $\left(\equiv G_{\text {imp, }, \mathrm{Z}}\right)$ are shown in fig. $4($ left $)$. Of course, systematic (non-perturbative) renormalization would be highly desirable [18].

\section{Physical interpretation}

The correlator $G_{\mathrm{E}}$ was computed at next-to-leading order (NLO) in continuum in ref. [19]. A comparison with our lattice results, improved and renormalized as explained above, is shown in fig. $\oiint$ (left). We observe a clear enhancement over the NLO prediction over a wide Euclidean time interval. Subtracting the two results, one could in principle attempt a model-independent analytic continuation of the non-perturbative surplus, for instance along the lines of ref. [20]. Unfortunately, at the current stage, our resolution is not sufficient $\left(\sim 10^{-3}\right)$ for this task. Therefore we resort to a rough model-dependent interpretation of the data in the following.

Most transport coefficients are related to quantities which are conserved in the free limit, and therefore arise in connection with a narrow transport peak. This yields a contribution to the Euclidean correlator which is almost constant in $\tau$. Since the full Euclidean correlator diverges at short distances and is essentially perturbative there, ratios such as $G_{\text {lat }} / G_{\mathrm{NLO}}$ should only be enhanced around the middle of the Euclidean time interval (cf. e.g. refs. [ 4 , 5]). This is clearly not the case in fig. $4($ left), and a narrow transport peak can be excluded, as expected [6, 19]. 

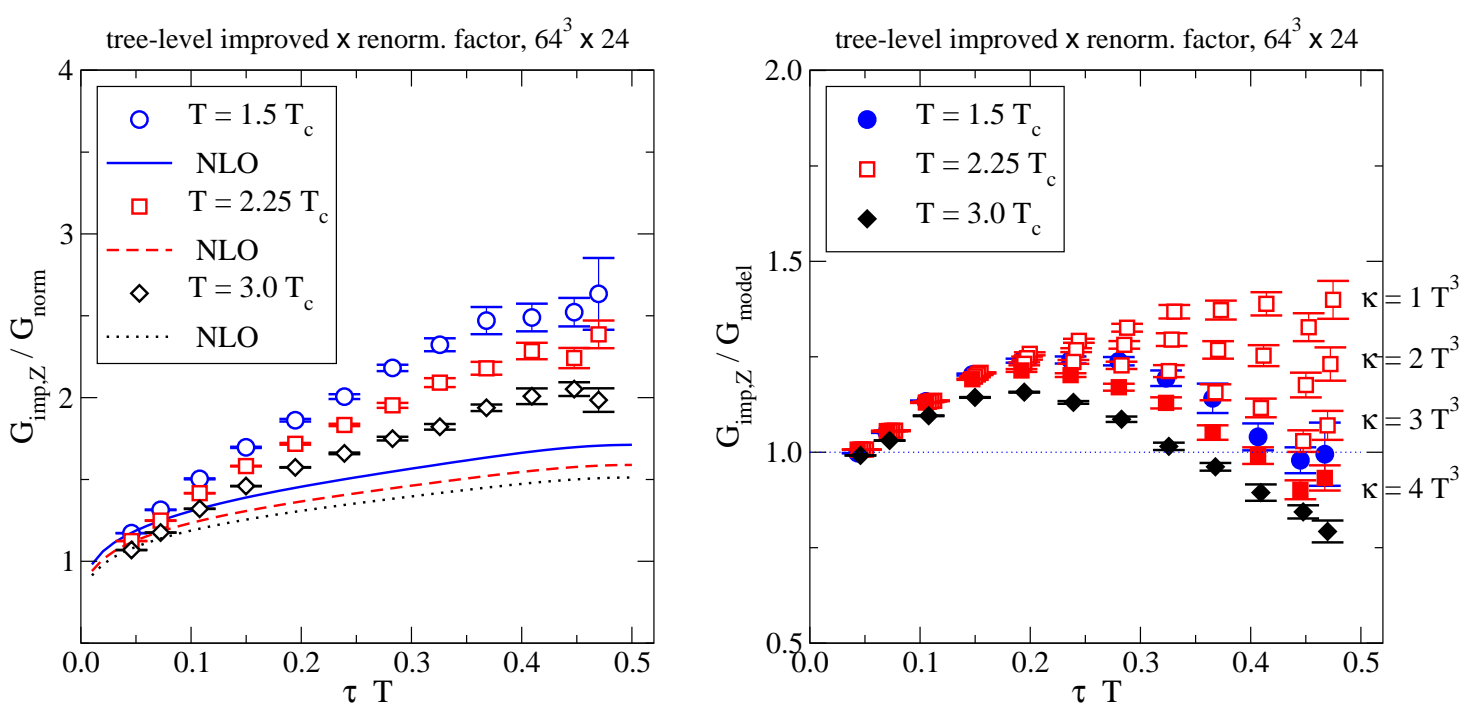

Figure 4: Left: Comparison of lattice with the NLO weak-coupling expansion [19]. Right: Like on the left but normalized to the model of eq. (6.1). Closed symbols correspond to $\kappa=4 T^{3}$, open ones to $T=2.25 T_{\mathrm{c}}$.

For a potentially more realistic model, we take inspiration from non-perturbative computations of the colour-electric correlator in other theories, one of them strongly coupled $\mathscr{N}=4$ Super-YangMills in the large- $N_{\mathrm{c}}$ limit [8, 21], another classical lattice gauge theory with $N_{\mathrm{c}}=3$ [22]. Both suggest $\rho_{\mathrm{E}}(\omega) / \omega$ to essentially flatten off below some frequency scale. Therefore, we define

$$
\rho_{\text {model }}(\omega) \equiv \max \left\{\rho_{\mathrm{NLO}}(\omega), \frac{\omega \kappa}{2 T}\right\},
$$

with the free parameter $\kappa$ representing directly the momentum diffusion coefficient according to eq. (2.2), and compute the corresponding Euclidean correlator from

$$
G_{\text {model }}(\tau) \equiv \int_{0}^{\infty} \frac{\mathrm{d} \omega}{\pi} \rho_{\text {model }}(\omega) \frac{\cosh \left(\frac{1}{2}-\tau T\right) \frac{\omega}{T}}{\sinh \frac{\omega}{2 T}} .
$$

Results are shown in fig. 4(right). We observe that despite extending its influence to larger frequencies than a narrow transport peak, the model does not capture the full shape of the Euclidean correlator; probably, extra "power" needs to be added to $\rho(\omega)$ at even larger frequencies.

Nevertheless, aiming at a match around the middle of the Euclidean time interval, we can read from fig. $₫$ (right) that $\kappa \sim 4 T^{3}$ at $T=1.5 T_{\mathrm{c}} ; \kappa \sim 3.5 T^{3}$ at $T=2.25 T_{\mathrm{c}}$; and $\kappa \sim 2.5 T^{3}$ at $T=3.0 T_{\mathrm{c}}$. The NLO weak-coupling expansion yields values $\kappa \sim 2 T^{3}[3]$ in this temperature range, and classical lattice gauge theory suggests that this could be an underestimate [22]. Converting $\kappa$ to the "usual" diffusion coefficient $D=2 T^{2} / \kappa$, we obtain $D \sim(0.5 \ldots 0.8) / T$, which might lie in a phenomenologically acceptable range (cf. e.g. refs. [8, 23]). In ref. [4], values $D \sim 0.3 / T$ were cited for the charm case. It is interesting that, despite the very rough nature of all of these estimates, a somewhat consistent picture appears to emerge.

\section{Conclusions}

As the results in fig. 4 show, it is possible to obtain results for the colour-electric correlator which unambiguously demand an enhancement of the non-perturbative interactions over the 
weak-coupling description and which furthermore strongly constrain the shape of the corresponding spectral function. Of course, for quantitative statements, careful infinite-volume and continuum extrapolations need to be taken, statistical uncertainties should be further reduced, and a more careful modelling of the spectral shape is called for.

\section{Acknowledgements}

We thank H. Meyer for providing us with lattice data from ref. [7] for comparison. A.F. was partly supported by the DFG International Graduate School Quantum Fields and Strongly Interacting Matter, and M.L. and J.L. by the BMBF under the project Heavy Quarks as a Bridge between Heavy Ion Collisions and QCD.

\section{References}

[1] A. Dainese [ALICE Collaboration], 1106.4042.

[2] G.D. Moore and D. Teaney, Phys. Rev. C 71 (2005) 064904 [hep-ph/0412346].

[3] S. Caron-Huot and G.D. Moore, JHEP 02 (2008) 081 [0801.2173].

[4] H.T. Ding, A. Francis, O. Kaczmarek, F. Karsch, H. Satz and W. Söldner, 1107.0311.

[5] H.T. Ding et al, Phys. Rev. D 83 (2011) 034504 [1012.4963].

[6] S. Caron-Huot, M. Laine and G.D. Moore, JHEP 04 (2009) 053 [0901.1195].

[7] H.B. Meyer, New J. Phys. 13 (2011) 035008 [1012.0234].

[8] J. Casalderrey-Solana and D. Teaney, Phys. Rev. D 74 (2006) 085012 [hep-ph/0605199].

[9] H.B. Meyer, Eur. Phys. J. A 47 (2011) 86 [1104.3708].

[10] G. Parisi, R. Petronzio and F. Rapuano, Phys. Lett. B 128 (1983) 418.

[11] P. de Forcrand and C. Roiesnel, Phys. Lett. B 151 (1985) 77.

[12] M. Lüscher and P. Weisz, JHEP 09 (2001) 010 [hep-lat/0108014].

[13] H.B. Meyer, Phys. Rev. D 76 (2007) 101701 [0704.1801].

[14] R. Sommer, Nucl. Phys. B 411 (1994) 839 [hep-lat/9310022].

[15] H.B. Meyer, JHEP 06 (2009) 077 [0904.1806].

[16] K. Symanzik, Nucl. Phys. B 226 (1983) 187.

[17] E. Eichten and B. Hill, Phys. Lett. B 240 (1990) 193.

[18] R. Sommer, 1008.0710.

[19] Y. Burnier, M. Laine, J. Langelage and L. Mether, JHEP 08 (2010) 094 [1006.0867].

[20] Y. Burnier, M. Laine and L. Mether, Eur. Phys. J. C 71 (2011) 1619 [1101.5534].

[21] S.S. Gubser, Nucl. Phys. B 790 (2008) 175 [hep-th/0612143].

[22] M. Laine, G.D. Moore, O. Philipsen and M. Tassler, JHEP 05 (2009) 014 [0902.2856].

[23] H. van Hees, M. Mannarelli, V. Greco and R. Rapp, Phys. Rev. Lett. 100 (2008) 192301 [0709.2884]. 